BULL. AUSTRAL. MATH. SOC.

$65 \mathrm{R} 20$

VOL. $24(1981), 153-154$.

$45 B 05,45 L 05$

\title{
THE NUMERICAL SOLUTION OF \\ FREDHOLM INTEGRAL EQUATIONS \\ OF THE SECOND KIND
}

IVAN G. GRAHAM

This thesis tackles some problems encountered in the numerical solution of Fredholm integral equations of the second kind. We are concerned specifically with the applicability and numerical performance of algorithms for these equations, and are guided by the existence of the following problems.

(i) Theoretically, the applicability of many algorithms often depends on certain highly abstract assumptions being satisfied. These assumptions are often difficult to verify in practice.

(ii) Error analyses for certain algorithms have tended to assume that the given information and the solution are smooth, and hence predict a higher order of convergence than that obtained in practice (where there are usually singularities present).

In Chapter 2 we develop practical methods for deciding whether a given integral operator is compact as an operator between certain spaces of functions. This solves a problem of type (i), since compactness is an abstract assumption used in the analysis of many algorithms for integral equations. In Chapter 3 we look at a class of weakly singular convolution type equations (typical of many that arise in practice), and answer the question: What kind of singularities arise in the solutions to such equations? In Chapter 4, the results of Chapter 3 are used to give a

Received 3 April 1981. Thesis submitted to the University of New South Wales, October 1980. Degree approved, March 1981. Supervisor: Professor I.H. Sloan. 
realistic error analysis (that is, one which takes account of the singularities in kernel and solution) for Galerkin type methods for the class of equations introduced in Chapter 3 , hence solving a problem of type (ii) for that class. The results of Chapters 3 and 4 concern only one dimensional integral equations. An analysis of collocation methods for two dimensional equations is given in Chapter 5 . Convergence rates are obtained for the cases of equations with both smooth kernels and weakly singular kernels. The analysis in the latter case depends on a characterisation of the properties of the solution to a typical two dimensional weakly singuiar equation. This characterisation is also given in Chapter 5. The methods proposed in Chapter 5 are illustrated in Chapter 6 by the numerical solution of a two dimensional equation arising in electrical engineering.

School of Mathematics, University of New South Wales, PO Box 1 , Kensington, New South Wales 2033, Australia. 